\title{
Integración arquitectónica de sistema fotovoltaico en escuela estatal rural de Santiago del Estero, Argentina
}

Gabriela Giuliano*, Beatriz Garzón **

\section{RESUMEN}

El artículo expone una alternativa de integración arquitectónica de un sistema fotovoltaico autónomo, en una escuela estatal rural de la provincia de Santiago del Estero-Argentina; con el objetivo de lograr generación eléctrica no convencional y ahorro energético, para el funcionamiento y la sostenibilidad del hábitat educativo. Los resultados alcanzados fueron: 1 ) análisis de costos de energía eléctrica, 2) dimensionamiento de un sistema fotovoltaico autónomo, 3) diseño y estudio detallado de la instalación. En conclusión, se determinó que con un diseño tecnológico sustentable integrado a la arquitectura, es posible crear un espacio funcional de usos múltiples, para el aprovechamiento de toda la comunidad educativa.

Palabras claves: Escuela Rural, Sistema Fotovoltaico, Integración Arquitectónica.

\section{ABSTRACT}

The article exposes an alternative of architectural integration of an autonomous photovoltaic system to a rural state school of the province of Santiago del EsteroArgentina, with the objective of achieving unconventional electricity generation and energy saving for the operation and sustainability of the educational habitat. The results achieved were: 1) analysis of electricity costs, 2) dimensioning of an autonomous photovoltaic system, 3) design and detailed study of the installation. In conclusion, it was determined that with a sustainable technological design integrated to the architecture, it is possible to create a functional space of multiple uses for the use of the ent $i-$ re educational community.

Keywords:

Rural School, Photovoltaic System, Architectural Integration.

*CONICET, ITA, CESPER, FCEyT- UNSE. Av. Belgrano (S) No 1912, CP (4200), Santiago del Estero, Argentina, e-mail: gm.giuliano@gmail.com.

* CONICET, FAU-SCAIT, UNT. Av. Nestor Kirchner No 1900, CP (4000), San Miguel de Tucumán, Tucumán, Argentina. 


\section{INTRODUCCIÓN}

El presente trabajo se inscribe, en el marco de un acuerdo de colaboración mutua entre CONICET y la Dirección General de Arquitectura de Santiago del Estero (DGA) -institución estatal responsable de la producción y mantenimiento de escuelas en la provincia- con el propósito de diseñar escuelas bioclimáticas, con uso racional de la energía convencional y la incorporación de energías renovables. Según el último censo nacional de infraestructura escolar (CENIE, 2014), en la provincia sobre un total de 1.463 escuelas estatales relevadas, 622 establecimientos cuentan con provisión de energía eléctrica a través de red, 144 utilizan grupo electrógeno y el resto de los establecimientos escolares no cuentan con acceso. Por otro lado, la implementación del "Proyecto de Energías Renovables en Mercados Rurales" (PERMER), desde 2001 a 1a actualidad, impactó únicamente en 165 escuelas rurales, con obras de sistemas fotovoltaicos autónomos (SFA), que involucran su instalación, solo sobre estructura soporte, sin contemplar su integración arquitectónica. Sobre esta base, surge la necesidad de comenzar a generar propuestas de soluciones alternativas.

\section{OBJETIVOS}

1) Analizar aspectos geográficos-climáticos y arquitectónicos-tecnológicos del prototipo escolar.

2) Estimar consumos y costos actuales de energía eléctrica de fuente fósil.

3) Proponer una alternativa para la disminución del consumo en iluminación.
4) Diseñar un sistema fotovoltaico autónomo con integración arquitectónica.

\section{METODOLOGÍA}

Se utilizó una combinación de metodologías para la presente investigación: analítica, descriptiva, correlacional y aplicada. Los resultados alcanzados fueron: 1) Se consideraron las condicionantes geográficas y climáticas de la localidad;2) Se realizó un análisis arquitectónico-tecnológico del prototipo, 3) Se evaluaron los costos de iluminación actuales; 4) Se efectuó una propuesta de mejora de artefactos de iluminación para disminuir el consumo; 5) Se dimensionó un SFAS, en función de valores de radiación solar y de consumo propuestos; 6) Se diseñaron detalles para su instalación, logrando su integración arquitectónica.

\section{RESULTADOS}

1) Definición de condicionantes geográficas y climáticas

La escuela $\mathrm{N}^{\circ} 653$ es un establecimiento educativo estatal de nivel primario, que se ubica en zona rural (Fig. No 1) de la localidad del Fisco de Fátima, perteneciente al departamento Jiménez, provincia Santiago del Estero. La localización corresponde a Zona Biambiental IIb, - Cálida, Subzona con amplitud Térmica menor a $14^{\circ} \mathrm{C}$ (Fig. No 2 ), según norma IRAM 11.603-2012. E1 clima es "seco semiárido estepario", según clasificación de Kôppen (1936), el mismo se caracteriza por un período estival muy cálido con las altas temperaturas y lluvioso entre los meses de octubre a marzo, en cambio la época invernal es seca y registra temperaturas bajas. 

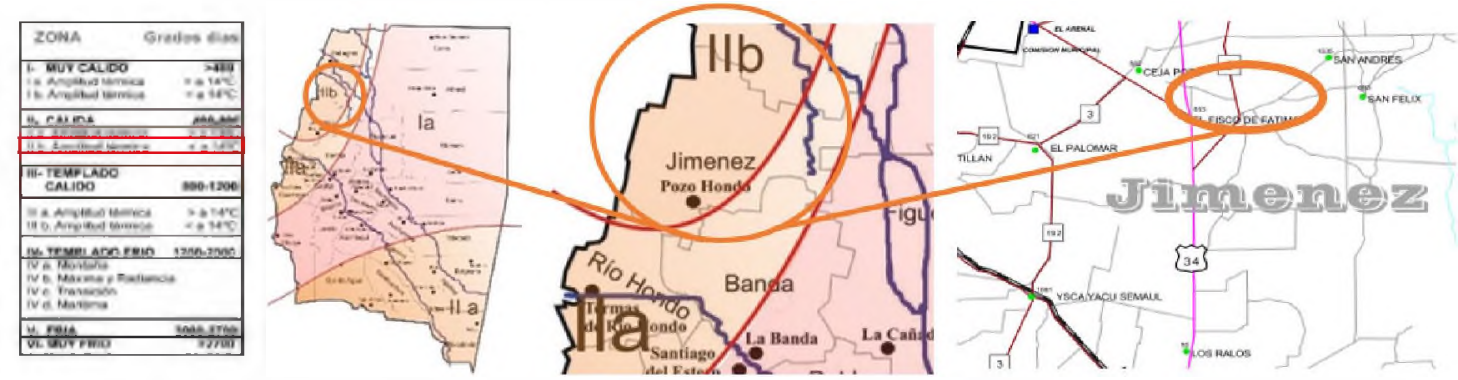

Figura Nº 1: Santiago del Estero, Zona Bioclimática "Cálida IIb”" (Norma IRAM 11.603-2012).

2) Análisis arquitectónico- tecnológico de la escuela

El edificio escolar, es de perímetro libre y se implanta hacia el norte, en el primer medio del terreno (Fig. No 3 y 4). El partido arquitectónico es lineal, con eje mayor en sentido "NE-NO". La escuela tiene 3 aulas con uso intensivo, una cuarta y quinta

que sirven a modo de depósito, cuenta además con cocina, sanitarios y dirección. En el terreno, se encuentran plantados árboles en forma planificada, a modo de cortina de vientos y de protección del asoleamiento, que arrojan sombra en paramentos y techo, quedando liberado de sombras arrojadas, durante la mañana solo el sector del acceso.
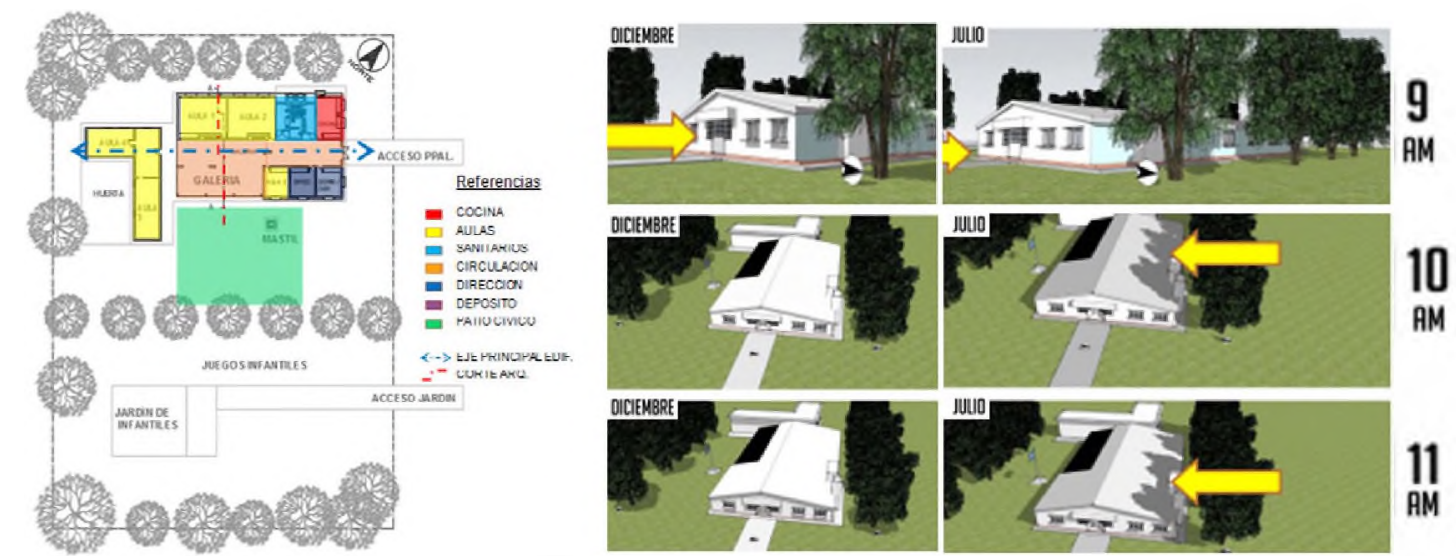

Figura $\mathrm{N}^{\circ}$ 2: Planta con zonificación funcional - Figura Nª 3: Plantación arbórea y sombras arrojadas

3) Evaluación de costos de iluminación según consumos actuales

La escuela está provista con electricidad de Red. En el interior, la instalación eléctrica es completa y embutida. La iluminación interior existente, es de lámparas tipo fluorescentes tubo y compactas, y la exterior, se resuelve con lámparas tipo alógenas (Fig.
No 4- Tabla No 1). La iluminación artificial total, es responsable de un $55 \%$ del consumo total energético diario (Wh/día). En la Figura No 5, puede observarse los consumos de cada bimestre, verificándose un incremento del costo de la energía eléctrica, que en el transcurso de 2 años, fue de $545 \%$ aproximadamente. Con ese ritmo sostenido de au- 
mentos, el estado provincial pagará cada año costos más onerosos por la energía eléctrica. Por tal motivo, se propone la reducción del consumo de iluminación, mediante tecnología LED y el diseño de un sistema fotovoltaico autónomo, para abastecer a toda la escuela (SFA).

4) Propuesta de reducción del consumo energético eléctrico
Debido a que en los 2 últimos años, se incrementó considerablemente del costo de la energía eléctrica en la provincia, con la tendencia de continuar acrecentándose. Se propone la disminución del consumo, mediante el reemplazo de las lámparas convencionales de bajo consumo, por lámparas del tipo LED (Tabla No 2- Fig. No 7).

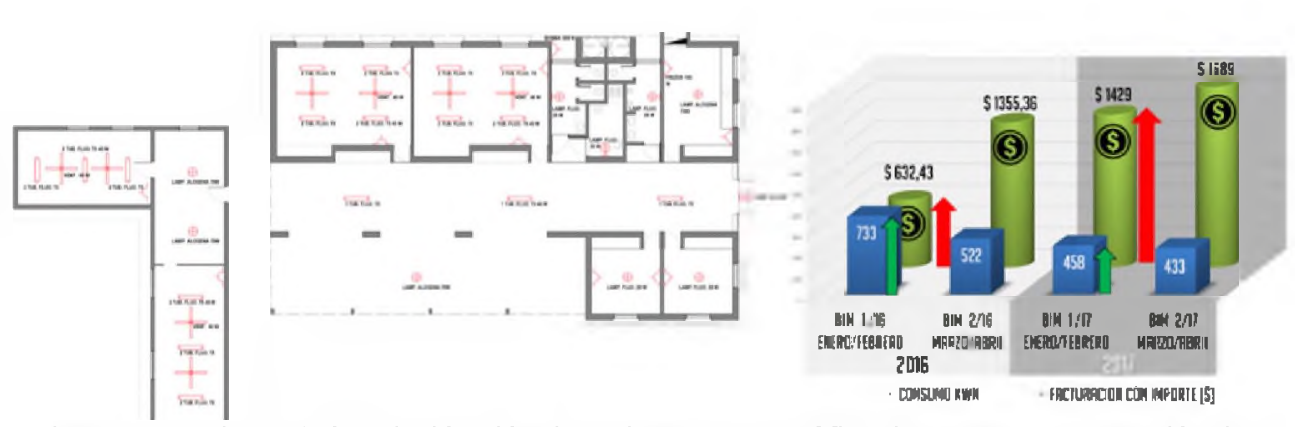

Figura $N^{\circ}$ 4: Planta de instalación eléctrica, Figura $N^{\circ} 5$ : Gráfico de consumo y costo eléctrico.

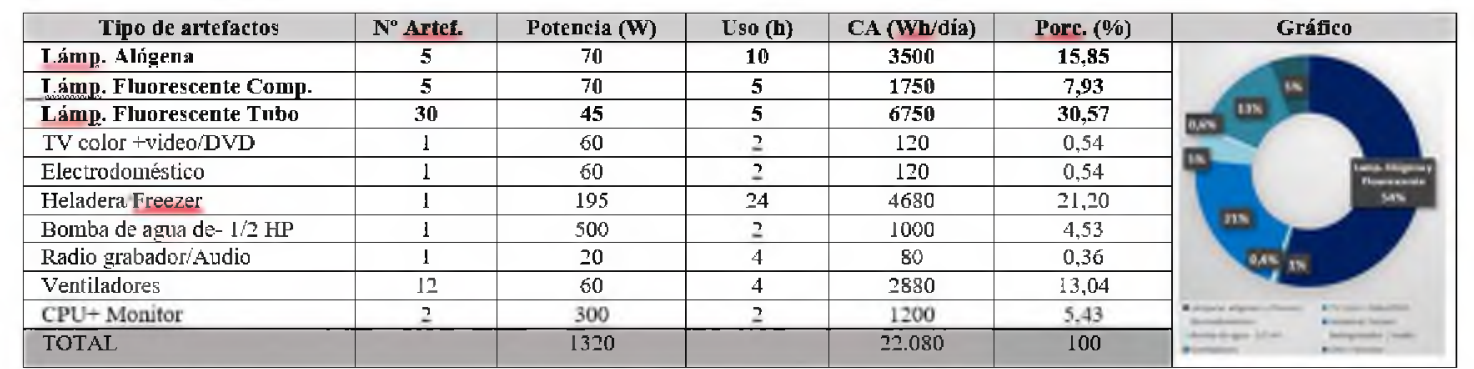

Tabla $\mathrm{N}^{\circ}$ 1: Consumo de eléctrico (Wh/día), Figura $\mathrm{N}^{\circ}$ 6: Gráfico de consumo eléctrico (\%)

\begin{tabular}{|c|c|c|c|c|c|c|}
\hline Tipo de artefactos & $\mathbf{N}^{\circ}$ Artef. & Potencia (W) & Uso (b) & CA (Wh/dia) & Porc. (\%) & Gráfico \\
\hline Lámpara LED & 9 & 11 & 4 & 396 & 2,99 & \\
\hline Lámpara Tubo LED & 32 & 26 & 4 & 3328 & 25,17 & \\
\hline TV color +video/DVD & 1 & 60 & 2 & 120 & 0.91 & \\
\hline Electrodomésticos & 1 & 60 & 2 & 120 & 0,91 & a \\
\hline Heladera Freezer & 1 & 195 & 24 & 4680 & 35,39 & \\
\hline Bomba de agua de- $1 / 2 \mathrm{HP}$ & 1 & 210 & 2 & 420 & 3,18 & 무 \\
\hline Radio grabador/Audio & 1 & 20 & 4 & 80 & 0.60 & \\
\hline Ventiladores & 12 & 60 & 4 & 2880 & 21.78 & \\
\hline CPU+ Monitor & 2 & 300 & 2 & 1200 & 9.07 & \\
\hline TOTAL & & 936 & & 13.224 & 100 & $=$ \\
\hline
\end{tabular}

Tabla $\mathrm{N}^{\mathrm{0}}$ 2: Consumo propuesto (Wh/día), Figura $\mathrm{N}^{\mathrm{v}}$ 7: Gráfico propuesta de consumo (\%). 
5) Dimensionado del sistema fotovoltaico autónomo simple

Mediante el calculador "FotovArq" (Giuliano y Garzón, 2017) se dimensionó un SFAS: el número de módulos FV, sus dimensiones y su integración arquitectóni- ca. El método utilizado, fue el de "amperios horas", tomando valores de consumo y de radiación solar particulares de la localidad donde se ubica el edificio escolar. Además se adoptaron otros valores, pertinentes para el cálculo (Tablas No 3 y 4).

\begin{tabular}{|l|l|c|c|}
\hline \multicolumn{1}{|c|}{ Designación } & Datos generales & Valores & Unidades \\
\hline$\eta$ & Días de Autonomía & 5 & días \\
\hline$V$ & Tensión Nominal Batería & 24 & $\mathrm{~V}$ \\
\hline$W_{p p}$ & Potencia Pico de cada Módulo & 260 & $\mathrm{~W}$ \\
\hline$\eta_{c}$ & Pérdidas de Conexión y Dispersión de parámetros & 0,1 & \\
\hline$\eta_{G}$ & Eficiencia de carga y descarga de la batería & 0,9 & \\
\hline$P_{a}$ & Máxima Profundidad de Descarga & 0,7 & \\
\hline$l_{i x v}$ & Eficiencia del inversor & 0,9 & \\
\hline Radiación diaria total & Radiación diaria total & 2.5 & $\mathrm{KWh} / \mathrm{m}^{2}$ \\
\hline
\end{tabular}

Tabla N 3 : Inicio de carga de datos en el programa calculador "FotovArq"

\begin{tabular}{|c|c|c|c|}
\hline Cálculos & Formulas & Resultados & Unidades \\
\hline Carga Total (Lt) & 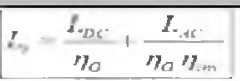 & 16.324 & $\mathrm{Wh} / \mathrm{dia}$ \\
\hline Capacidad de Batería (CB) & $C B=\frac{C B^{*}}{V}=\frac{L \|}{V p_{d} \eta_{c}}$ & 48.589 & Ah. \\
\hline Horas Solar P1co $\left(\mathrm{HSP}_{\beta}\right)$ & 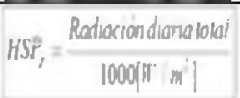 & 2,5 & $\mathrm{~h}$ \\
\hline Números de Paneles $\left(\mathrm{N}^{\circ} \mathrm{P}\right)$ & $V^{n}=\frac{l}{\pi_{1} / A S P_{p}\left(1-n_{1}\right)}$ & 28 & Módulos \\
\hline
\end{tabular}

Tabla $\mathrm{N}^{\circ}$ 4: Procedimiento de cálculo: determinación de Lt, CB, $\mathrm{HSP}_{\beta}$ y $\mathrm{N}^{\circ} \mathrm{P}$.

En el programa FotovArq, se dimensio- string "cadenas", para maximizar la colección nó el sistema con un panel FV denominado solar y lograr un diseño eficiente integrado P-260 W, de 1,62 m2 de superficie total; y arquitectónicamente, útil a diversas activipermite estimar la superficie de la totalidad dades de la comunidad educativa (Tabla $5 \mathrm{y}$ de los módulos y la distancia mínima entre Fig. No 8).

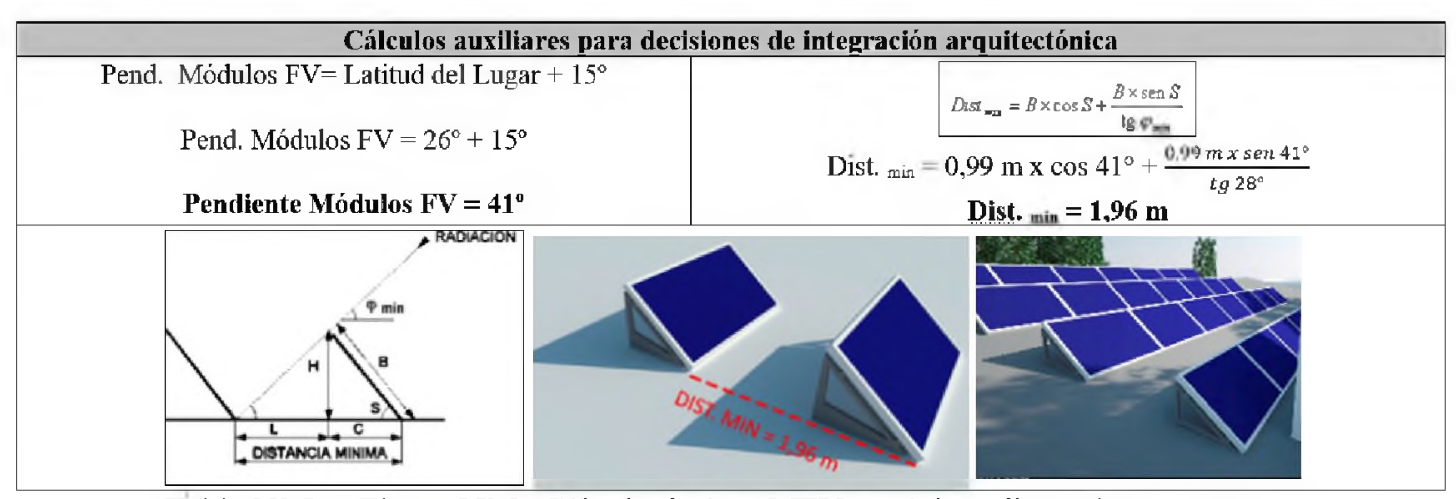

Tabla $\mathrm{N}^{\circ} 5$ y Figura $\mathrm{N}^{\circ}$ 8: Cálculo de área MFV y mínima distancia entre string. 
6) Diseño de detalles para la integración arquitectónica:

La propuesta de integración arquitectónica del SFA, es la de construir una estructura soporte del mismo, de modo que cumpla al mismo tiempo, con la función de cubrir un Salón de Usos Múltiples (SUM). E1 resultado del diseño, estuvo en función de la búsqueda de alternativas para optimización de la captación de la radiación solar en verano e invierno (Garzón et al., 2017). Fue seleccionada la alternativa No3, donde el SUM se ubica en el sector izquierdo del acceso, evitando la sombra arrojada de la vegetación arbórea existente (Figuras No 9 a 12).
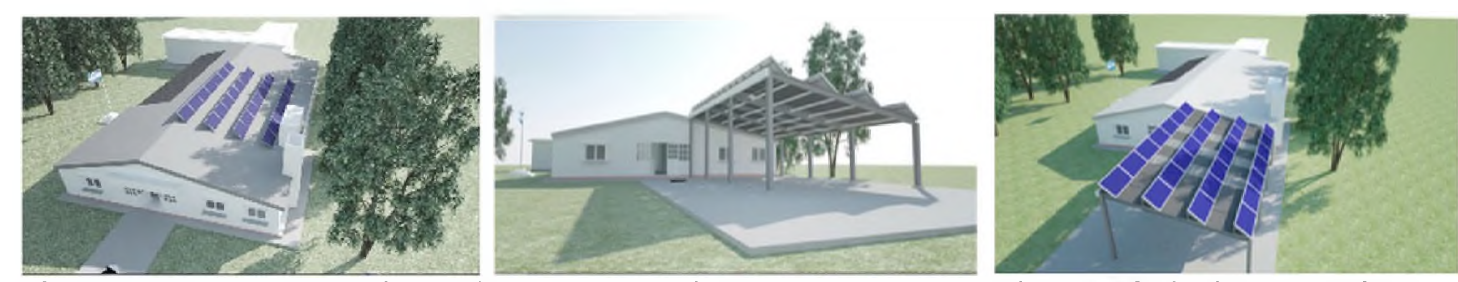

Figuras $\mathrm{N}^{\circ}$ 9, 10 y 11 : Alternativas 1 y 2: Techo y S.U.M. con sombra arrojada de vegetación

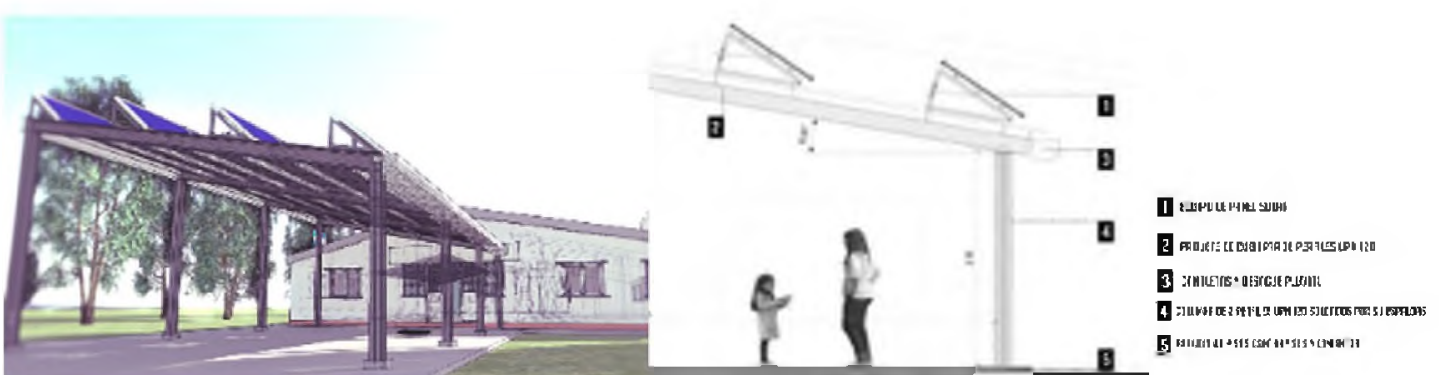

Figuras $\mathrm{N}^{\circ}$ 12: Alternativa 3-Seleccionada por mayor eficiencia. Figl 13: Detalle Instalación

\section{CONCLUSIÓN}

Se determinó que en la escuela estatal rural No 653 , el sistema de iluminación artificial interior, tiene un $55 \%$ de influencia en el consumo total de la energía eléctrica. Debido a los incrementos sostenidos, de las tarifas de energía eléctrica a nivel provincial, y con tendencia a continuar acrecentándose, es que se propuso la reducción del consumo con la incorporación de lámparas tipo LED; con lo cual, se logró que la incidencia del ítem, se reduzca a un $28 \%$ del total. Se dimensionó, el SFA para abastecer la totalidad de la demanda de energía eléctrica de la institución educativa. Se logró conciliar, el uso de tecnología sustentable para el aprovechamiento de la energía solar, con un diseño integral de adecuación arquitectónica, que satisface la necesidad de un espacio de múltiple función, para las actividades de la comunidad rural de la localidad del Fisco de Fátima en particular; y en general, con el aporte de pautas para potenciar la sustentabilidad del hábitat escolar rural. En una futura etapa de trabajo, se prevé coordinar 
entre CONICET- DGA y el PERMER; con la intención de articular trabajo para fortalecer la promoción de la integración arquitectónica, de sistemas fotovoltaicos autónomos a instalar.

\section{REFERENCIAS}

Giuliano G. Garzón, B.; (2017). "FotovArq": Programa de Cálculo de Sistemas Fotovoltaicos autónomos y su integración Arquitec- tónica. Obra inscripta en la DNDA, Formulario No 232.930, CABA, Noviembre de 2017.

Garzón, B.; Giuliano G.; Maldonado J. (2017). "Desarrollo, evaluación y transferencia de estrategias y disposiciones arquitectónicas y tecnológicas no convencionales con uso racional y renovable de la energía para la sustentabilidad y salubridad del Hábitat". Julio 2017, PPA- UNT- FAU. 